\section{Diálogos sobre o processo de trabalho em saúde bucal no Brasil: uma análise com base no PMAQ-AB}

\author{
Dialogues on the work process in oral health in \\ Brazil: an analysis based on the PMAQ-AB survey
}

\author{
Discusiones sobre el proceso de trabajo en el \\ ámbito de la salud bucal en Brasil: un análisis \\ basado en el PMAQ-AB
}

\author{
Daniela Malagoni Fagundes 1 \\ Erika Barbara Abreu Fonseca Thomaz 2 \\ Rejane Christine de Sousa Queiroz 2 \\ Thiago Augusto Hernandes Rocha 3 \\ Núbia Cristina da Silva 3 \\ Joao Ricardo Nickenig Vissoci 4 \\ Maria Cristina Marino Calvo 5 \\ Luiz Augusto Facchini ${ }_{6}$
}

\section{Resumo}

Os objetivos do estudo foram: (1) descrever o processo de trabalho das equipes de saúde bucal (ESB) do Brasil, com base nos atributos essenciais da atenção primária à saúde, segundo regiões, tipo de equipe e características socioeconômicas dos municípios; e (2) verificar se os dados do processo de trabalho das ESB do Programa Nacional de Melhoria do Acesso e da Qualidade da Atenção Básica (PMAQ-AB) foram capazes de aferir tais atributos. Estudo ecológico, de abrangência nacional, com dados do ciclo I do PMAQ-AB. Foram feitas análises descritivas, fatoriais exploratória e confirmatória $(\alpha=5 \%)$. Os construtos formados foram analisados à luz dos atributos essenciais da atenção primária à saúde (primeiro contato, coordenação do cuidado, integralidade e longitudinalidade). Formaram-se os três primeiros construtos e um quarto fator, denominado ações em prótese dentária. Porém, o atributo longitudinalidade não foi conformado. As medidas de ajuste dos modelos foram satisfatórias. As cargas fatoriais foram maiores que 0,5, exceto para duas variáveis do fator 3. As ações mais realizadas pelas ESB (> 60\%) foram as do primeiro contato e as menos comuns foram as da integralidade, destacandose ter referência para especialidades $(7,6 \%)$. Houve diferenças no processo de trabalho das ESB entre as regiões, tipo de equipe e estrato de certificação ( $p<$ 0,05 ). Conclui-se que os dados de processo de trabalho das ESB do ciclo I do $P M A Q-A B$ foram capazes de discriminar três dos quatro atributos essenciais da atenção primária à saúde na rotina dos serviços. Sugere-se aprofundar a avaliação da longitudinalidade. Ademais, as ESB participantes do ciclo I do $P M A Q-A B$ precisam avançar nas ações relacionadas à integralidade e coordenação do cuidado.

Avaliação em Saúde; Serviços de Saúde; Atenção Primária à Saúde; Saúde Bucal

\author{
Correspondência \\ E. B. A. F. Thomaz \\ Departamento de Saúde Pública, Universidade Federal do \\ Maranhão. \\ Rua Barão de Itapary 155, São Luís, MA 65020-070, Brasil. \\ ebthomaz@gmail.com \\ 1 Rede Nordeste de Formação em Saúde da Família, São Luís, \\ Brasil. \\ 2 Universidade Federal do Maranhão, São Luís, Brasil. \\ 3 Universidade Federal de Minas Gerais, Belo Horizonte, Brasil. \\ ${ }_{4}$ Duke University, Durham, Estados Unidos. \\ 5 Universidade Federal de Santa Catarina, Florianópolis, Brasil. \\ 6 Universidade Federal de Pelotas, Pelotas, Brasil.
}




\section{Introdução}

A Estratégia Saúde da Família (ESF) é referência para um modelo assistencial que prioriza ações de proteção e promoção de saúde dos indivíduos e famílias 1, incluindo ações de saúde bucal 2. Está fundamentada nos princípios do Sistema Único de Saúde (SUS) e segue os atributos da atenção primária à saúde, propostos por Starfield 3. Esses atributos têm como princípios ordenadores (essenciais) o primeiro contato, a longitudinalidade, a integralidade e a coordenação do cuidado; e como princípios derivados, a centralização na família, a orientação comunitária e a competência cultural. Logo, o cumprimento dos atributos da atenção primária à saúde constitui valioso indicador para o processo de avaliação da qualidade dos serviços ofertados 4 .

Estudos sobre as práticas de saúde bucal apresentam resultados contraditórios. Alguns identificam avanços no modelo assistencial 5,6,7,8, enquanto outros referem a manutenção das práticas tradicionais 6,9,10,11,12,13. Tais avaliações, entretanto, são baseadas em amostras locais e com diferentes instrumentos avaliativos.

Com o propósito de institucionalizar a cultura de avaliação da atenção em saúde e induzir mudanças nos serviços, o Ministério da Saúde realizou, entre 2011 e 2013, o primeiro ciclo do Programa Nacional de Melhoria do Acesso e da Qualidade da Atenção Básica (PMAQ-AB). Uma das fases do PMAQ-AB é a avaliação externa, que inclui avaliação do processo de trabalho das equipes de saúde bucal (ESB) 14. Os instrumentos de coleta de dados foram construídos utilizando a Política Nacional de Atenção Básica (PNAB) 15 como referencial teórico. Esse documento referencia os atributos essenciais da atenção primária à saúde, propostos por Starfield 3, como eixos organizativos da atenção básica no Brasil. Portanto, o redirecionamento do modelo assistencial brasileiro, centrado na atenção primária à saúde, está diretamente condicionado ao cumprimento de tais atributos no processo de trabalho das ESB.

O presente estudo tem por objetivo: (1) descrever o processo de trabalho das ESB do Brasil, com base nos atributos essenciais da atenção primária á saúde, segundo regiões, tipo de equipe da atenção primária à saúde e características socioeconômicas dos municípios; e (2) verificar se os dados do processo de trabalho das ESB do ciclo I do PMAQ-AB foram capazes de aferir tais atributos.

\section{Material e métodos}

Trata-se de um estudo ecológico, de abrangência nacional, com dados secundários, provenientes da avaliação externa das unidades básicas de saúde (UBS) do ciclo I do PMAQ-AB, desenvolvido pelo Ministério da Saúde em parceria com várias instituições de ensino e pesquisa do país, de 2012 a 2013. As unidades de análise foram as ESB.

Das 32.337 equipes de atenção básica implantadas no território brasileiro, $17.482(54,1 \%)$ aderiram ao ciclo I do PMAQ-AB, tendo sido analisados dados de 17.202 equipes de atenção básica. As perdas (280 equipes, 1,6\%) foram decorrentes de recusas, ausência do profissional na unidade, mesmo após várias tentativas, ou impossibilidade de deslocamento dos avaliadores até a unidade de saúde. Desse total, 12.075 eram ESF com saúde bucal (representando 45,22\% do total de 26.705 equipes da ESF com saúde bucal identificadas na ocasião) e 328 eram equipes parametrizadas com saúde bucal (representando 9,76\% do total de 3.360 equipes parametrizadas com saúde bucal identificadas). Dessa forma, 12.403 ESB participaram do ciclo I do PMAQ-AB, distribuídas em 3.965 municípios, sendo 811 ESB em municípios do Norte do país, 4.755 no Nordeste, 3.930 no Sudeste, 1.982 no Sul e 925 no Centro-oeste. Esse mesmo tamanho amostral foi incluído na presente investigação.

Foram utilizados dados referentes ao processo de trabalho das ESB, o que corresponde a 19 questões (Quadro 1), respondidas pelas equipes que aderiram ao ciclo I, em entrevistas realizadas na UBS com os profissionais das equipes, preferencialmente o cirurgião dentista. Com essas variáveis, realizou-se análise fatorial exploratória (AFE) para a identificação dos construtos (fatores) referentes aos atributos essenciais da atenção primária à saúde. Os atributos estão descritos no Quadro 2 16,17,18,19.

Foram estimadas as cargas fatoriais padronizadas (CFP) da covariância entre cada variável e o fator construído. CFP acima de 0,7 indicavam boa validade convergente e abaixo de 0,95 indicavam boa validade discriminante. 
Quadro 1

Variáveis relacionadas ao processo de trabalho das equipes de saúde bucal. PMAQ-AB, 2012-2013.

\begin{tabular}{|c|c|c|}
\hline Variável & Pergunta & Categorias de resposta \\
\hline v1 & Como são agendadas as consultas em saúde bucal? & $\begin{array}{l}\text { Qualquer dia da semana em qualquer } \\
\text { horário; qualquer dia, em horários } \\
\text { específicos; } 4 \text { ou mais dias específicos } \\
\text { fixados na semana; até } 3 \text { dias específicos } \\
\text { fixos; ou outro }\end{array}$ \\
\hline v2 & A equipe faz todos os procedimentos básicos de saúde bucal? * & Sim ou não \\
\hline v3 & Existe acolhimento à demanda espontânea específica para saúde bucal? & Sim ou não \\
\hline v4 & $\begin{array}{l}\text { O profissional realiza avaliação de risco e vulnerabilidade no primeiro } \\
\text { atendimento? }\end{array}$ & Sim ou não \\
\hline v5 & A oferta é definida em função do risco identificado? & Sim ou não \\
\hline v6 & A ESB utiliza algum protocolo de acolhimento à demanda espontânea? & Sim ou não \\
\hline v7 & $\begin{array}{l}\text { A equipe garante agenda } \mathrm{p} / \text { a continuidade do tratamento de quem iniciou } \\
\text { tratamento? }\end{array}$ & Sim ou não \\
\hline v8 & $\begin{array}{l}\text { A equipe realiza campanhas p/ detecção de lesões suspeita de câncer de boca e } \\
\text { encaminha casos de câncer de boca? }\end{array}$ & Sim ou não \\
\hline v9 & $\begin{array}{l}\text { A equipe registra e acompanha os casos suspeitos/confirmados de câncer de } \\
\text { boca? }\end{array}$ & Sim ou não \\
\hline v10 & Existe CEO de referência para a sua equipe? & Sim ou não \\
\hline v11 & O município possui referência para quantas das especialidades obrigatórias? ** & $0,1,2,3,4$ ou 5 \\
\hline v12 & Número de protocolos de fluxo de encaminhamento. ${ }^{\star \star \star}$ & $0,1,2,3$ ou 4 \\
\hline v13 & $\begin{array}{l}\text { A ESB promove ações p/ identificação de pessoas que necessitam de prótese } \\
\text { dentária? }\end{array}$ & Sim ou não \\
\hline v14 & A ESB realiza a moldagem da prótese dentária na Unidade de Saúde? & Sim ou não \\
\hline v15 & A equipe possui referência para profissional protesista? & Sim ou não \\
\hline v16 & A ESB realiza entrega da prótese e acompanhamento do usuário? & Sim ou não \\
\hline v17 & Existe Laboratório de Prótese Dentária no município? & Sim ou não \\
\hline v18 & Qual é o tipo de prótese realizada no laboratório? \# & $0,1,2$ ou 3 \\
\hline v19 & $\begin{array}{l}\text { Após solicitado pelo profissional da Atenção Básica, qual tempo médio, em dias, } \\
\text { de espera em cada especialidade? ** }\end{array}$ & $0,1,2,3,4$ e 5 \\
\hline
\end{tabular}

CEO: Centro de Especialidades Odontológicas; ESB: equipes de saúde bucal.

Fonte: Instrumento de Avaliação Externa, Módulo II, Ciclo I do PMAQ-AB, 2012-2013.

* Aplicação de selante, aplicação tópica de flúor, restauração de amálgama, restauração de resina composta, exodontia, selamento provisório de cavidade dentária, curativo de demora, drenagem de abscesso dento-alveolar, raspagem, alisamento e polimento supragengivais;

** Endodontia, periodontia, cirurgia bucomaxilofacial, estomatologia, outra;

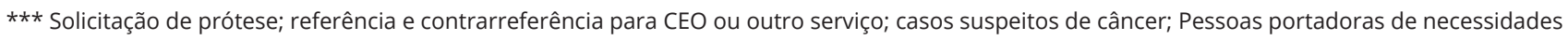
especiais;

\# Total, parcial removível e fixa.

Inicialmente, foram testados modelos com diferentes números de fatores, identificando aquele com os mais adequados índices de ajuste, considerando-se: qui-quadrado $\left(\chi^{2}\right)$; erro médio quadrático de aproximação (Root Mean Square Error of Approximation - RMSEA); limite superior do intervalo de confiança de 90\% do RMSEA; índices incrementais Comparative Fit Index (CFI) e Tucker-Lewis Index (TLI); e Weighted Root Mean Square Residual (WRMR). Os modelos foram considerados bem ajustados quando o valor do $\chi^{2}$ foi maior que 0,05 ; RMSEA menor que 0,5 ; limite superior do IC90\% do RMSEA menor que 0,08; CFI e TLI maiores que 0,90; e WRMR menor que 1,0 20. 


\section{Quadro 2}

Atributos essenciais da atenção primária à saúde.

\begin{tabular}{|c|c|}
\hline Primeiro contato & $\begin{array}{l}\text { Definido por Souza } 16 \text { segundo dois aspectos: (1) ausência de barreiras que dificultem o acesso da população, como a } \\
\text { localização da unidade de saúde e o horário de funcionamento; e (2) reconhecimento do serviço pela população como } \\
\text { seu primeiro recurso para o atendimento a uma necessidade em saúde. Para Starfield 17, implica a acessibilidade e o } \\
\text { uso de serviços para cada novo problema para os quais se procura atenção à saúde. }\end{array}$ \\
\hline Integralidade & $\begin{array}{l}\text { Um dos pilares na construção do SUS consagrado pela Constituição Federal de } 1988 \text { e possui quatro dimensões: (1) } \\
\text { primazia das ações de promoção e prevenção; (2) atenção nos três níveis de complexidade da assistência médica; } \\
\text { (3) articulação das ações de promoção, proteção e prevenção; e (4) abordagem integral do indivíduo e das famílias } \\
\text { 18. Pressupõe, portanto, a existência de uma rede de serviços articulada em distintos níveis de complexidade e de } \\
\text { competências. Para Souza 16, é a capacidade de os serviços oferecerem um conjunto de ações para atender aos } \\
\text { problemas mais comuns identificados na população. Implica que as unidades de atenção primária à saúde devem } \\
\text { fazer arranjos para que o paciente receba todos os tipos de serviços de atenção à saúde 17,18. Desse atributo deriva } \\
\text { o cuidado integral - capacidade da equipe de saúde em lidar com as necessidades em saúde do indivíduo, da } \\
\text { família ou das comunidades, seja resolvendo (em } 85 \% \text { das situações) ou referindo aos outros pontos de atenção à } \\
\text { saúde (cuidados secundários, terciários ou outros setores como educação, saneamento, habitação etc.) } 19 \text {. Assim, } \\
\text { integralidade considera um conceito amplo de saúde, em que necessidades básicas biopsicossociais, culturais e } \\
\text { subjetivas são reconhecidas. }\end{array}$ \\
\hline $\begin{array}{l}\text { Coordenação do } \\
\text { cuidado }\end{array}$ & $\begin{array}{l}\text { Pressupõe o acompanhamento da atenção ao usuário 17. Diz respeito às formas de relacionamento dos serviços de } \\
\text { atenção primária à saúde com os demais pontos de atenção disponíveis na rede prestadora16,18. É essencial para o } \\
\text { sucesso dos demais atributos da atenção primária á saúde e, para que ocorra, é necessário haver disponibilidade e } \\
\text { utilização de dados } 19 .\end{array}$ \\
\hline Longitudinalidade & $\begin{array}{l}\text { Aporte regular de cuidados pela equipe de saúde. Podendo ser traduzida como o vínculo, a responsabilização, a } \\
\text { "relação mútua" entre o usuário e o profissional de saúde e a continuidade de oferta regular dos serviços } 17 .\end{array}$ \\
\hline
\end{tabular}

No modelo selecionado na AFE, identificaram-se quais variáveis estavam relacionadas com cada fator, considerando as maiores CFP. Em seguida, levou-se esse modelo para a análise fatorial confirmatória (AFC). Testou-se o modelo com os fatores de primeira ordem previamente identificados e, ainda, um outro modelo com a inclusão de um fator de segunda ordem (denominado processo de trabalho das ESB) formado pelos fatores de primeira ordem. Nessa etapa, foram analisadas as CFP, variância residual e coeficiente de determinação $\left(\mathrm{R}^{2}\right)$ para as variáveis de cada fator. Usou-se o estimador WLSMV (Robust DWLS Approach), com parametrização Theta.

$\mathrm{Na}$ AFC, para acessar a validade convergente, foram calculadas as variâncias médias extraídas (Average Variance Extracted - AVE) e as confiabilidades compostas (Composite Reliability - CR) para cada fator. Valores acima de 0,5 foram considerados aceitáveis e acima de 0,7 muito bons 21,22.

Para a AFE e AFC, foi utilizado o pacote estatístico Mplus, versão 7.0 (Muthén \& Muthén, Los Angeles, Estados Unidos), adotando-se o nível de 5\% de significância.

Todas as variáveis mantidas no modelo após a AFC foram sumarizadas por meio de frequências absolutas e percentuais, segundo região geopolítica do país (Norte, Nordeste, Sul, Sudeste, Centro-oeste), tipo de equipe (ESF com saúde bucal ou parametrizada com saúde bucal 22) e estrato de certificação do PMAQ-AB (1 a 6), onde o 1 representa o estrato de municípios com pior situação socioeconômica e menor porte populacional; e o 6, melhor situação socioeconômica e maior porte populacional. Para a construção desse índice, foram considerados cinco indicadores, conforme definição do Ministério da Saúde 15: Produto Interno Bruto (PIB) per capita; percentual da população com plano de saúde; percentual da população com bolsa família; percentual da população em extrema pobreza; e densidade demográfica. O software Stata 12.0 (StataCorp LP, College Station, Estados Unidos) foi utilizado nas análises descritivas.

O estudo foi submetido ao Comitê de Ética em Pesquisa da Faculdade de Medicina/Universidade Federal de Pelotas, em cumprimento aos requisitos exigidos pela Resolução no 196/1996, 
posteriormente substituída pela Resolução no 466/2012, do Conselho Nacional de Saúde, tendo sido aprovado segundo Ofício 38/12 de 10 de maio de 2012, com parecer número 38/2012.

\section{Resultados}

\section{Análise fatorial exploratória (AFE)}

Das 19 perguntas do processo de trabalho das ESB do PMAQ-AB (Quadro 1), as variáveis v14 ("a equipe de saúde bucal realiza a moldagem da prótese dentária na unidade de saúde") e v19 ("tempo médio de espera para o atendimento especializado") foram excluídas da AFE devido à alta correlação com outras variáveis do modelo.

$\mathrm{O}$ modelo que reteve quatro fatores obteve o melhor ajuste: valor de $\mathrm{p}<0,001$; RMSEA $=0,023$; IC90\%: 0,022-0,025; CFI = 0,987; TLI = 0,976; MRFV = 0,686 (Tabela 1). O fator 1 (f1: variáveis v3, v4 e v5) correspondeu a um construto que denominamos primeiro contato. O fator 2 (f2: variáveis v10, v11, v12 e v18) foi denominado integralidade. O fator 3 (f3: variáveis v1, v2, v6, v7, v8 e v9) foi denominado coordenação do cuidado. O fator 4 (f4: variáveis v13, v15, v16 e v17) conformou um construto que se denominou ações em prótese dentária. Todas as CFP ficaram acima de 0,30, com exceção de v1 ("como são agendadas as consultas em saúde bucal") e v18 ("qual é o tipo de prótese realizada no laboratório"). A variável v1 foi mantida nas análises, mas v18 foi excluída (Figura 1). A manutenção de v1 deveu-se à sua importância teórica e, ainda, ao fato de que a sua exclusão prejudicava no ajuste do modelo.

As variáveis v1, v2, v7 e v8 geraram cargas cruzadas em f1 ef3, de forma significante, demonstrando que poderiam contribuir para a construção de fatores distintos. Cada variável foi alocada apenas para um fator e os melhores indicadores de ajuste do modelo foram considerados para tal definição.

Quanto a f1 e f4, todas as CFP foram superiores a 0,5 , indicando boa validade convergente. Porém, v4 (no f1) e v15 (no f4) apresentaram CFP maiores que 0,9, indicando problemas de validade

\section{Tabela 1}

Medidas de ajuste nas análises fatorial exploratória e confirmatória. Brasil, 2012-2013.

\begin{tabular}{|c|c|c|c|c|c|c|}
\hline \multirow[t]{2}{*}{ Índices de ajuste } & \multicolumn{4}{|c|}{ Análise fatorial exploratória } & \multicolumn{2}{|c|}{ Análise fatorial confirmatória } \\
\hline & 1 fator & 2 fatores & 3 fatores & 4 fatores & $\begin{array}{l}\text { Modelo com fatores de } \\
\text { 1a ordem }\end{array}$ & $\begin{array}{c}\text { Modelo com } \\
\text { fatores de 1a e 2a } \\
\text { ordem }\end{array}$ \\
\hline $\begin{array}{l}\text { Número de parâmetros } \\
\text { livres }\end{array}$ & 20 & 36 & 51 & 65 & 41 & 39 \\
\hline Graus de liberdade & 119 & 103 & 88 & 74 & 98 & 100 \\
\hline$\chi^{2 *}$ & $11.988,82$ & $4.149,15$ & $2.103,87$ & 764,37 & $3.272,21$ & $5.155,62$ \\
\hline Valor de $\mathrm{p}$ do $\chi^{2}$ & $<0,001$ & $<0,001$ & $<0,001$ & $<0,001$ & $<0,001$ & $<0,001$ \\
\hline RMSEA ** & 0,076 & 0,048 & 0,036 & 0,023 & 0,043 & 0,054 \\
\hline IC90\% do RMSEA *** & $0,075-0,077$ & $0,047-0,049$ & $0,035-0,038$ & $0,022-0,025$ & $0,042-0,045$ & $0,053-0,055$ \\
\hline $\mathrm{CFI} \#$ & 0,775 & 0,923 & 0,962 & 0,987 & 0,937 & 0,899 \\
\hline TLI \#\# & 0,743 & 0,899 & 0,941 & 0,976 & 0,922 & 0,879 \\
\hline WRMR \#\# & 6,1021 & 1,0376 & 0,4850 & 0,6860 & 4,116 & 5,245 \\
\hline
\end{tabular}

IC90\%: intervalo de 90\% de confiança; CFI: conparative fit index; RMSEA: Root Mean Square Error of Approximation; TLI: Tucker Lewis Index; WRMR: Weighted Root Mean Square Residual.

* Teste qui-quadrado (referência: menor valor);

** Referência: menor que 0,05);

*** Referência: limite superior do IC90\% menor que 0,08;

\# Referência: maior que 0,9;

\#\# Referência: maior que 0,9. 
Figura 1

Cargas fatoriais padronizadas e resíduos, na análise fatorial confirmatória, incluindo um fator de 2ạ ordem. Brasil, 2012-2013.

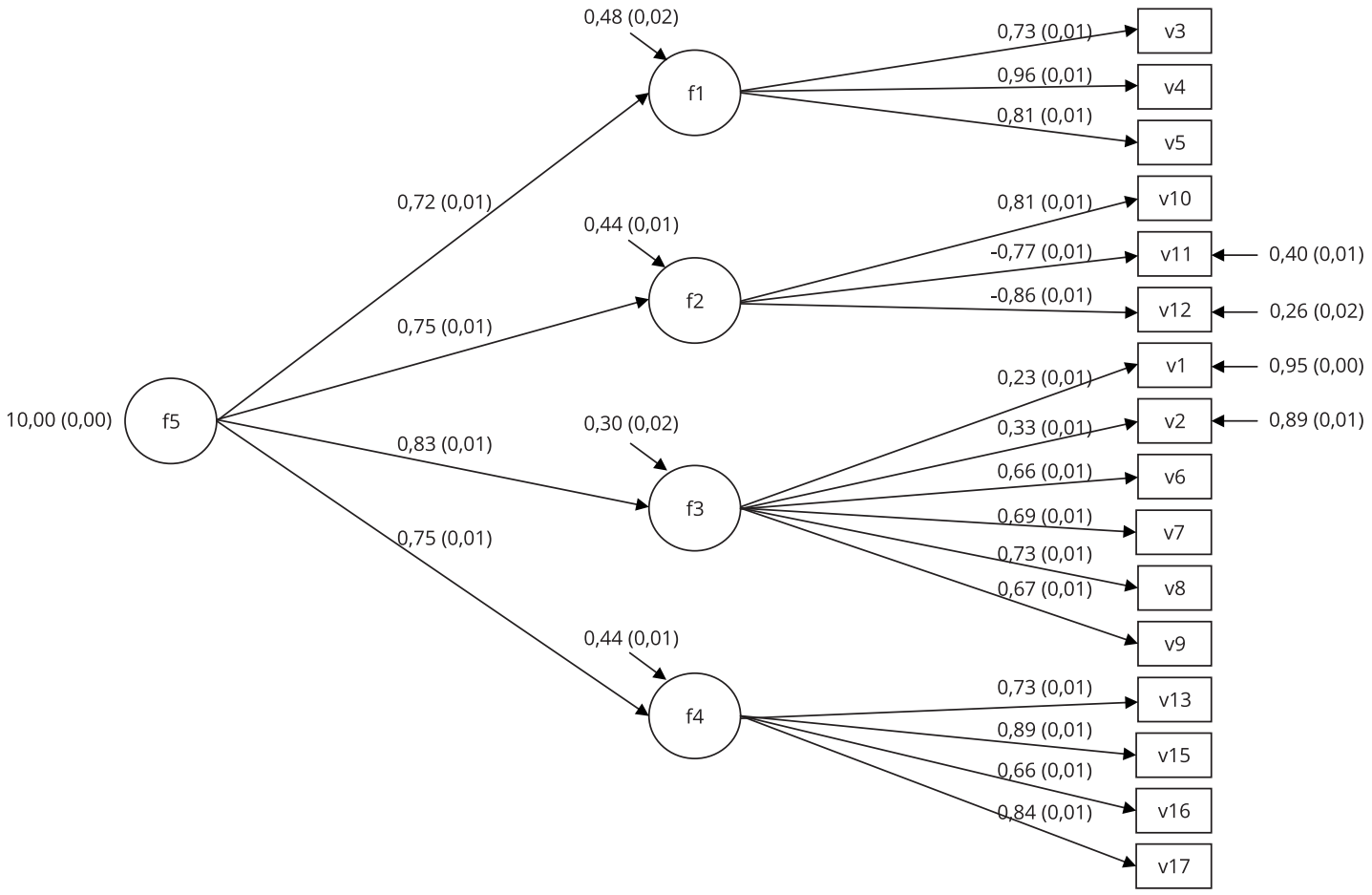

CEO: Centro de Especialidades Odontológicas; ESB: equipe de saúde bucal; f1: primeiro contato; f2: integralidade; f3: coordenação do cuidado; f4: ações em prótese dentária; f5: processo de trabalho das EBS.

v1: como são agendadas as consultas em saúde bucal? v2: a equipe faz todos os procedimentos básicos de saúde bucal? v3: existe acolhimento à demanda espontânea específica para saúde bucal? v4: o profissional realiza avaliação de risco e vulnerabilidade no $1^{\circ}$ atendimento? v5: a oferta é definida em função do risco identificado? v6: a ESB utiliza algum protocolo de acolhimento à demanda espontânea? v7: a ESB garante continuidade do trat. de quem iniciou? v8: A ESB realiza campanhas para detecção de câncer de boca e encaminha os casos suspeitos? v9: a ESB registra e acompanha casos de câncer de boca? v10: existe CEO de referência para a sua equipe? v11: o município possui referência para quantas especialidades obrigatórias? v12: número de protocolos de fluxo de encaminhamento. v13: a ESB faz ações de identificação de pessoas para prótese dentária? v14: a ESB realiza a moldagem da prótese dentária na unidade básica de saúde? v15: a equipe possui referência para profissional protesista? v16: a ESB faz entrega da prótese dentária e acompanhamento do usuário? v17: existe laboratório de prótese dentária no município?

discriminante. O f3 apresentou duas CFP menores que 0,5 (v1 e v2), revelando problemas de validade convergente; mas todas as cargas fatoriais foram abaixo de 0,95 , demonstrando boa validade discriminante (dados não tabulados).

\section{Análise fatorial confirmatória (AFC)}

$\mathrm{Na}$ AFC, testou-se o modelo com quatro fatores, apontado pela AFE como o de melhor ajuste. Analisou-se se os 16 itens (após exclusão dos itens v14, v18 e v19) do instrumento de avaliação do processo de trabalho das ESB realmente mediam os atributos essenciais da atenção primária à saúde e se eles conformavam um quinto fator (f5), que denominamos processo de trabalho das ESB.

A análise foi realizada em duas etapas: na primeira, considerando-se os quatro fatores acima definidos (modelo 1); e na segunda, acrescentando-se um fator de segunda ordem (f5: processo de trabalho) (modelo 2). 
Os parâmetros do modelo 1 (sem o f5) indicaram melhor ajuste que o modelo 2 (com o f5): menor $\chi^{2}(3272,21$ versus 5155,62$)$; menor RMSEA $(0,043$ versus 0,054$)$; menor limite superior do IC90\% $(0,045$ versus 0,055$)$; maior CFI $(0,937$ versus 0,899$)$; maior TLI $(0,922$ versus 0,879$)$; e menor WRMR $(4,116$ versus 5,245$)$. Isso sugere que as variáveis do questionário do PMAQ-AB explicam parcialmente o processo de trabalho das ESB (Tabela 1).

Ambos os modelos apresentaram CFP altas (acima de 0,50), exceto para v1 e v2, com cargas moderadas (em torno de 0,30 ), mas todas significantes (Figura 1). Os coeficientes de determinação ( $\left.\mathrm{R}^{2}\right)$ também foram altos (acima de 0,5) para a maioria das variáveis, indicando bom valor preditivo na construção dos fatores. O f3 teve os menores valores de $\mathrm{R}^{2}$.

Os valores de AVE e CR foram, respectivamente, 0,70 e 0,88 (f1), 0,66 e 0,41 (f2), 0,35 e 0,75 (f3) e 0,62 e 0,86 (f4). Tais valores indicam boa validade convergente, especialmente para of 1 e f4. As covariâncias $(\mathrm{cv})$ entre os fatores foram acima de 0,5 e significantes $(\mathrm{p}<0,01)$, indicando baixa validade discriminante (f1 e f3, $\mathrm{cv}=0,78$; f2 e f3, $\mathrm{cv}=0,57$; $\mathrm{f} 2$ e f4, $\mathrm{cv}=0,69$; e f 3 e f4, $\mathrm{cv}=0,56$ ).

Ao incluir no modelo um fator de 2a ordem, verificou-se que as medidas de ajuste do modelo ficaram piores, particularmente o CFI e TLI (Tabela 1). No entanto, todas as CFP relacionadas a f5 ficaram acima de 0,70 e com coeficientes de determinação $\left(R^{2}\right)$ acima de 0,50 e significantes $(p<0,001)$ (Figura 1).

\section{Análise do processo de trabalho segundo atributos essenciais da atenção primária à saúde}

As ações e serviços que configuraram o primeiro contato (f1) foram referidas por mais de $60 \%$ das ESB avaliadas. Porém, verificou-se baixo percentual de realização das ações relacionadas aos demais fatores, sobretudo os que definiram a integralidade (f2) e as ações em prótese dentária (f4). Os piores resultados foram encontrados para a existência de "referência para as cinco especialidades obrigatórias dos Centros de Especialidades Odontológicas (CEO)" (7,6\%) e para a "entrega e acompanhamento de próteses dentárias pelas ESB" (10,2\%). Das 16 ações listadas na Figura 2, apenas cinco foram realizadas por mais da metade das ESB brasileiras (Figura 2).

As equipes da região Nordeste que aderiram ao ciclo I do PMAQ-AB foram as que mais realizaram o primeiro contato (f1). A integralidade (f2), coordenação do cuidado (f3) e as ações em prótese dentária (f4) foram pouco observadas em todo o país, sendo realizadas por menos de $50 \%$ das equipes em todas as regiões, especialmente no Norte.

As ESB vinculadas à ESF foram as que mais trabalhavam em conformidade com os atributos essenciais da atenção primária à saúde avaliados neste estudo, embora apresentando ainda baixos percentuais de realização de ações e serviços relacionados à integralidade e coordenação do cuidado. Apenas o percentual de "agendamento em qualquer dia e horário da semana" foi maior nas equipes parametrizadas $(39,8 \%)$ do que nas ESF (36,3\%). As ESB pertencentes aos municípios mais pobres e de menor porte populacional (estrato 1) realizavam mais ações de primeiro contato (f1) e coordenação do cuidado (f3). Verificou-se maior proporção de ações relacionadas à integralidade (f2) nos municípios mais desenvolvidos (Tabela 2).

\section{Discussão}

Os dados processados pelos instrumentos de coleta de dados do PMAQ-AB não identificaram todos os atributos essenciais da atenção primária à saúde no primeiro ciclo do programa. Os dados expressaram três dos quatro atributos essenciais da atenção primária definidos por Starfield 3 - primeiro contato, integralidade e coordenação do cuidado; contudo não o atributo longitudinalidade.

Este é o primeiro estudo de que se tem conhecimento que utilizou AFE e AFC na análise do instrumento de avaliação externa do ciclo I do PMAQ-AB referente ao processo de trabalho das ESB da atenção básica do SUS. Os dados evidenciaram bons índices de ajuste do modelo, além de CFP altas e significantes $(\mathrm{p}<0,05)$, especialmente nos construtos primeiro contato, integralidade e ações em prótese. $\mathrm{O}$ fato indica a formação de bons construtos, capazes de expressar adequadamente o que se propõem. No entanto, o constructo coordenação do cuidado apresentou uma variável com CFP menor que 0,3 (v1: “Como são agendadas as consultas em saúde básica?"). Essa variável poderia ter 
Figura 2

Caracterização (\%) do processo de trabalho das equipes de saúde bucal (ESB) no Brasil, 2012-2013.

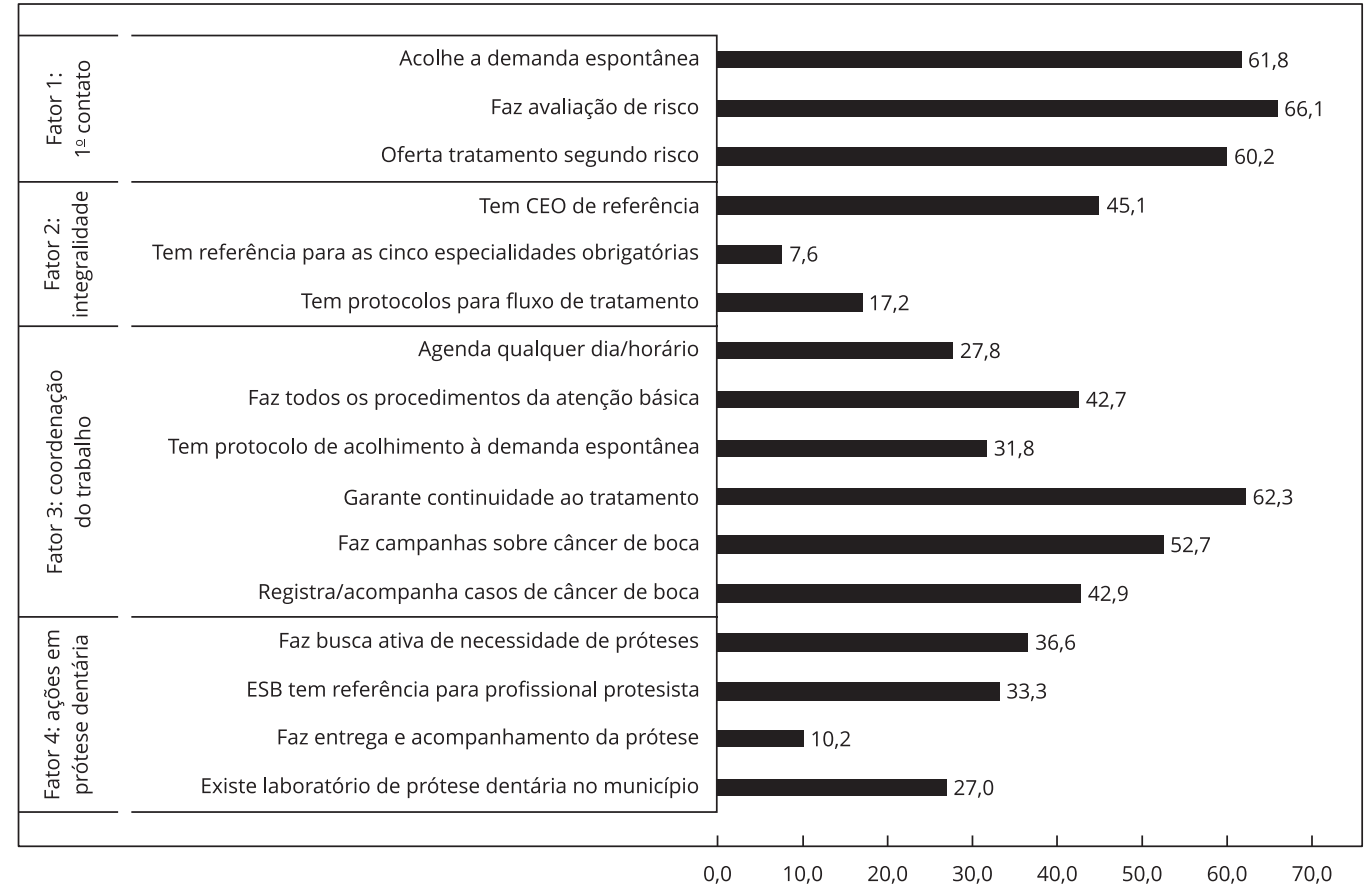

Fonte: banco de dados PMAQ-AB, ciclo I 2012-2013.

CEO: Centro de Especialidades Odontológicas.

sido removida do modelo. Entretanto, reduziria a qualidade do ajuste e, do ponto de vista teórico (Quadro 2), é importante na formação do fator. Por isso, foi mantida.

Atualmente, a análise de componentes principais (ACP) tem sido bastante empregada na construção e validação de instrumentos de coleta de dados. Tanto a ACP quanto a AF podem ser utilizadas para extrair fatores subjacentes; e envolvem procedimentos matemáticos que transformam um número de possíveis variáveis correlacionadas em um menor número de variáveis não correlacionadas (ACP) ou correlacionadas (AF). No entanto, embora sejam muito similares, possuem algumas diferenças: (1) em ACP, assume-se que toda a variabilidade de um item deve ser utilizada na análise, enquanto na $\mathrm{AF}$, define-se a priori o número de fatores que se quer extrair, admitindo-se variância residual; (2) na ACP, o objetivo é explicar o máximo possível de variância total nas variáveis, enquanto, na AF, é explicar as covariâncias ou correlações entre as variáveis; e (3) a ACP é especialmente indicada para reduzir os dados a um número menor de componentes, enquanto a $\mathrm{AF}$ é indicada para entender os construtos subjacentes aos dados 23. Portanto, considerando que os construtos formados não estão livres de erro randômico e que o objetivo do estudo é explicar correlações entre variáveis e examinar a estrutura dos dados, optou-se, nesta investigação, pela AFE seguida de AFC 24.

Destaca-se a formação de um fator que não caracteriza um atributo essencial - ações em prótese dentária. Desde a Política Nacional de Saúde Bucal (PNSB) 25, o Ministério da Saúde passou a encorajar que ações de moldagem e entrega de próteses fossem realizadas na atenção básica. Ainda que não seja um atributo essencial da atenção primária à saúde, essas ações foram priorizadas. Isso demonstra a preocupação do governo federal quanto ao problema do edentulismo no país. Pesquisas revelaram que a perda dentária precoce é um grave problema da população brasileira, e que a necessidade de algum tipo de prótese começa a partir dos 15 a 19 anos 26,27. Tal cenário pode ter motivado a inserção de variáveis relacionadas aos serviços de prótese dentária no instrumento do ciclo I do PMAQ-AB. 
Caracterização (n e \%) do processo de trabalho das equipes de saúde bucal (ESB), segundo região, tipo de equipe e estrato de certificação. Brasil, 2012-2013.

\begin{tabular}{|c|c|c|c|c|c|c|c|c|c|c|c|c|c|c|c|c|}
\hline & \multicolumn{3}{|c|}{$\begin{array}{c}\text { Fator } 1 \\
\text { Primeiro contato }\end{array}$} & \multicolumn{3}{|c|}{$\begin{array}{c}\text { Fator } 2 \\
\text { Integralidade }\end{array}$} & \multicolumn{6}{|c|}{$\begin{array}{c}\text { Fator } 3 \\
\text { Coordenação do cuidado }\end{array}$} & \multicolumn{4}{|c|}{$\begin{array}{c}\text { Fator } 4 \\
\text { Ações em prótese dentária }\end{array}$} \\
\hline & $\begin{array}{c}\text { Acolhe } \\
\text { a } \\
\text { deman- } \\
\text { da } \\
\text { espon- } \\
\text { tânea }\end{array}$ & $\begin{array}{c}\text { Faz } \\
\text { avali- } \\
\text { ação } \\
\text { de } \\
\text { risco }\end{array}$ & $\begin{array}{c}\text { Ofer- } \\
\text { ta } \\
\text { trata- } \\
\text { mento } \\
\text { se- } \\
\text { gun- } \\
\text { do } \\
\text { o } \\
\text { risco }\end{array}$ & $\begin{array}{c}\text { Tem } \\
\text { CEO } \\
\text { de } \\
\text { refe- } \\
\text { rên- } \\
\text { cia }\end{array}$ & $\begin{array}{l}\text { Tem } \\
\text { referê- } \\
\text { ncia } \\
\text { para } \\
\text { as } \\
\text { espe- } \\
\text { ciali- } \\
\text { dades } \\
\text { obri- } \\
\text { ga- } \\
\text { tórias }\end{array}$ & $\begin{array}{l}\text { Tem } \\
\text { proto- } \\
\text { colos } \\
\text { para } \\
\text { fluxo } \\
\text { de } \\
\text { trata- } \\
\text { mento }\end{array}$ & $\begin{array}{c}\text { Agen- } \\
\text { da } \\
\text { qual- } \\
\text { quer } \\
\text { dia/ } \\
\text { horá- } \\
\text { rio }\end{array}$ & $\begin{array}{c}\text { Faz } \\
\text { todos } \\
\text { os } \\
\text { pro- } \\
\text { cedi- } \\
\text { men- } \\
\text { tos } \\
\text { da } \\
\text { aten- } \\
\text { ção } \\
\text { básica }\end{array}$ & $\begin{array}{c}\text { Tem } \\
\text { proto- } \\
\text { colo } \\
\text { de } \\
\text { acolhi- } \\
\text { mento } \\
\text { à } \\
\text { deman- } \\
\text { da } \\
\text { espon- } \\
\text { tânea }\end{array}$ & $\begin{array}{c}\text { Ga- } \\
\text { rante } \\
\text { con- } \\
\text { tinui- } \\
\text { dade } \\
\text { do } \\
\text { trata- } \\
\text { men- } \\
\text { to }\end{array}$ & $\begin{array}{c}\text { Faz } \\
\text { cam- } \\
\text { pa- } \\
\text { nhas } \\
\text { sobre } \\
\text { cân- } \\
\text { cer } \\
\text { boca }\end{array}$ & $\begin{array}{l}\text { Regis- } \\
\text { tra/ } \\
\text { acom- } \\
\text { pa- } \\
\text { nha } \\
\text { casos } \\
\text { de } \\
\text { cân- } \\
\text { cer } \\
\text { boca }\end{array}$ & $\begin{array}{c}\text { Faz } \\
\text { busca } \\
\text { ativa } \\
\text { de } \\
\text { ne- } \\
\text { cessi- } \\
\text { dade } \\
\text { de } \\
\text { próte- } \\
\text { ses }\end{array}$ & $\begin{array}{c}\text { ESB } \\
\text { tem } \\
\text { refe- } \\
\text { rência } \\
\text { para } \\
\text { profis- } \\
\text { sio- } \\
\text { nal } \\
\text { prote- } \\
\text { sista }\end{array}$ & $\begin{array}{c}\text { Faz } \\
\text { entre- } \\
\text { ga } \\
\text { e } \\
\text { acom- } \\
\text { panha- } \\
\text { ment- } \\
\text { o } \\
\text { da } \\
\text { próte- } \\
\text { se }\end{array}$ & $\begin{array}{c}\text { Existe } \\
\text { Labo- } \\
\text { ra- } \\
\text { tório } \\
\text { de } \\
\text { pró- } \\
\text { te- } \\
\text { se } \\
\text { den- } \\
\text { tária }\end{array}$ \\
\hline \multicolumn{17}{|c|}{ Região } \\
\hline \multicolumn{17}{|c|}{ Norte } \\
\hline $\mathrm{n}$ & 649 & 704 & 633 & 478 & 23 & 98 & 254 & 309 & 313 & 669 & 469 & 318 & 307 & 260 & 54 & 222 \\
\hline$\%$ & 63,9 & 67,4 & 62,4 & 47,1 & 2,3 & 9,7 & 25,0 & 30,4 & 30,8 & 65,9 & 46,2 & 31,3 & 30,2 & 25,6 & 5,3 & 21,9 \\
\hline \multicolumn{17}{|c|}{ Nordeste } \\
\hline$n$ & 3.931 & 4.283 & 3.840 & 2.775 & 382 & 713 & 1.253 & 2.350 & 1.777 & 3.736 & 3.254 & 2.423 & 2.172 & 1.946 & 362 & 1.545 \\
\hline$\%$ & 71,1 & 77,0 & 69,5 & 50,2 & 6,9 & 12,9 & 22,6 & 42,5 & 32,1 & 67,6 & 58,9 & 43,8 & 39,3 & 35,2 & 6,5 & 28,0 \\
\hline \multicolumn{17}{|c|}{ Sudeste } \\
\hline $\mathrm{n}$ & 3.588 & 3.717 & 3.470 & 2.729 & 576 & 1.370 & 2.039 & 2.816 & 2.123 & 3.773 & 3.216 & 2.777 & 2.332 & 2.162 & 868 & 1873 \\
\hline$\%$ & 54,7 & 56,5 & 52,9 & 41,6 & 8,8 & 20,9 & 31,1 & 42,9 & 32,3 & 57,5 & 49,0 & 42,3 & 35,5 & 33,0 & 13,2 & 28,6 \\
\hline \multicolumn{17}{|l|}{ Sul } \\
\hline $\mathrm{n}$ & 1757 & 1849 & 1652 & 1293 & 250 & 552 & 852 & 1325 & 908 & 1718 & 1494 & 1382 & 1028 & 938 & 345 & 621 \\
\hline$\%$ & 60,3 & 63,3 & 56,7 & 44,3 & 8,6 & 18,9 & 29,2 & 45,4 & 31,1 & 58,9 & 51,2 & 47,4 & 35,2 & 32,2 & 11,8 & 21,3 \\
\hline \multicolumn{17}{|c|}{ Centro- } \\
\hline $\mathrm{n}$ & 699 & 814 & 752 & 486 & 65 & 219 & 379 & 550 & 350 & 814 & 627 & 481 & 463 & 425 & 129 & 370 \\
\hline$\%$ & 63,3 & 73,4 & 68,1 & 44,0 & 5,9 & 19,8 & 34,3 & 49,8 & 31,7 & 73,7 & 56,7 & 43,5 & 41,9 & 38,5 & 11,6 & 33,5 \\
\hline \multicolumn{17}{|c|}{ Tipo de } \\
\hline \multicolumn{17}{|c|}{$\begin{array}{l}\text { equipe } \\
\text { ESF }\end{array}$} \\
\hline $\mathrm{n}$ & 9.852 & 10.964 & 9.587 & 7.105 & 1.161 & 2.625 & 4.365 & 6.777 & 4.939 & 9.919 & 8.356 & 6.777 & 5.808 & 5.267 & 1.563 & 4.215 \\
\hline$\%$ & 81,9 & 90,8 & 79,7 & 59,1 & 9,6 & 21,8 & 36,3 & 56,3 & 41,1 & 82,5 & 69,5 & 56,3 & 48,3 & 43,6 & 13,0 & 34,9 \\
\hline \multicolumn{17}{|c|}{$\begin{array}{l}\text { Equipe } \\
\text { parame- } \\
\text { trizada }\end{array}$} \\
\hline $\mathrm{n}$ & 229 & 292 & 239 & 141 & 24 & 52 & 126 & 172 & 130 & 251 & 200 & 153 & 136 & 124 & 40 & 90 \\
\hline$\%$ & 72,4 & 89,0 & 75,6 & 44,6 & 7,3 & 16,4 & 39,8 & 54,4 & 41,1 & 79,4 & 63,2 & 48,4 & 43,0 & 37,8 & 12,6 & 27,4 \\
\hline \multicolumn{17}{|c|}{ Estrato } \\
\hline $\mathrm{n}$ & 1.497 & 1.645 & 1.514 & 766 & 69 & 179 & 750 & 1.048 & 634 & 1.599 & 1.261 & 1.010 & 844 & 634 & 346 & 178 \\
\hline$\%$ & 69,8 & 76,8 & 70,6 & 35,7 & 3,2 & 8,3 & 35,0 & 48,9 & 29,5 & 72,7 & 58,8 & 47,1 & 39,3 & 29,6 & 16,1 & 8,3 \\
\hline \multicolumn{17}{|l|}{2} \\
\hline $\mathrm{n}$ & 1.479 & 1.634 & 1.488 & 699 & 46 & 163 & 625 & 961 & 611 & 1.500 & 1.147 & 891 & 735 & 594 & 206 & 339 \\
\hline$\%$ & 65,4 & 72,4 & 65,8 & 30,9 & 2,0 & 7,2 & 27,6 & 42,5 & 27,0 & 66,4 & 50,7 & 39,4 & 32,5 & 26,3 & 9,1 & 15,0 \\
\hline \multicolumn{17}{|l|}{3} \\
\hline $\mathrm{n}$ & 1.752 & 1.858 & 1.714 & 1.058 & 107 & 294 & 645 & 1.053 & 790 & 1.733 & 1.381 & 1.027 & 980 & 798 & 169 & 642 \\
\hline$\%$ & 69,8 & 74,2 & 68,3 & 42,1 & 4,3 & 11,7 & 25,7 & 41,9 & 31,4 & 69,0 & 55,0 & 40,9 & 39,0 & 31,8 & 6,7 & 25,6 \\
\hline \multicolumn{17}{|c|}{ סו, נו } \\
\hline $\mathrm{n}$ & 2.173 & 2.306 & 2.127 & 1.587 & 205 & 535 & 1.116 & 1.532 & 1.035 & 2.198 & 1.864 & 1.532 & 1.315 & 1.250 & 334 & 982 \\
\hline$\%$ & 66,7 & 71,1 & 65,2 & 48,7 & 6,3 & 16,4 & 34,2 & 47,0 & 31,7 & 67,4 & 57,2 & 47,0 & 40,3 & 38,4 & 10,2 & 30,1 \\
\hline \multicolumn{17}{|l|}{5} \\
\hline $\mathrm{n}$ & 1.607 & 1.644 & 1.502 & 1.536 & 365 & 581 & 602 & 1.134 & 808 & 1.617 & 1.415 & 1.150 & 996 & 1.065 & 180 & 1.011 \\
\hline$\%$ & 57,5 & 60,1 & 53,7 & 54,9 & 13,1 & 20,8 & 21,5 & 40,6 & 28,9 & 57,8 & 50,6 & 41,1 & 35,6 & 38,1 & 6,4 & 36,2 \\
\hline 6 & & & & & & & & & & & & & & & & \\
\hline $\mathrm{n}$ & 2.116 & 1.529 & 2.002 & 2.115 & 504 & 1.200 & 1.039 & 1.622 & 1.593 & 2.103 & 1.992 & 1.771 & 1.432 & 1.390 & 523 & 1.479 \\
\hline$\%$ & 51,0 & 47,3 & 48,2 & 50,9 & 12,2 & 28,9 & 25,0 & 39,0 & 38,3 & 50,6 & 48,0 & 42,6 & 34,5 & 33,5 & 12,6 & 35,6 \\
\hline
\end{tabular}

CEO: Centro de Especialidades Odontológicas; ESF: Estratégia Saúde da Família. 
Ainda assim, os dados da presente investigação demonstram que as ações em prótese dentária ainda estão sendo pouco realizadas pelas ESB no Brasil. Menos de 50\% dos profissionais entrevistados no ciclo I do PMAQ-AB referiram incorporar tais ações em seus processos de trabalho.

Outras fragilidades no processo de trabalho das ESB do SUS foram identificadas. Parece que as ações e serviços em saúde bucal não estão sendo executados em conformidade com os atributos essenciais da atenção primária à saúde. Apenas as ações relacionadas ao primeiro contato (f1) estão sendo realizadas pela maioria das ESB no Brasil. Tais ações dizem respeito à redução de barreiras ao acesso da população, como o atendimento oportuno, baseado em critérios de risco e vulnerabilidade, bem como outras ações que aumentem a percepção, pela população, da UBS como o primeiro recurso para o atendimento às necessidades em saúde 16.

Ações relacionadas à integralidade (f2) ainda são pouco realizadas no país, mesmo tendo sido observado um aumento significativo da oferta de serviços odontológicos especializados com a criação dos CEO. Esse atributo depende da existência de uma rede de serviços articulada em distintos níveis de complexidade e de competências 16,17,18, consequentemente, ultrapassa os limites de governabilidade das ESB. A oferta das ações referentes à coordenação do cuidado (f3) foi bastante heterogênea, variando de $27,8 \%$ para o item "agendamento em qualquer dia/horário", a $62,3 \%$, para o item "garantia de continuidade do tratamento". Isso sugere que esse atributo ainda está em implantação no processo de trabalho das ESB brasileiras. Por sua vez, a longitudinalidade - traduzida como o aporte regular de cuidados pela equipe de saúde, gerando vínculo, responsabilização, e "relação mútua" entre o usuário e o profissional de saúde 17 -, não foi adequadamente mensurada pelo PMAQ-AB.

Uma variável (v7: "A equipe garante agenda para a continuidade do tratamento de quem iniciou tratamento?") foi a que mais se aproximou do conceito de longitudinalidade, mas não foi suficiente para medi-lo. Sugere-se que futuras versões do PMAQ-AB incorporem questões capazes de melhor avaliar o atributo no processo de trabalho das equipes de atenção básica.

Segundo Souza \& Roncalli 28 , a reorganização das ações na atenção básica pautadas numa nova concepção do processo saúde-doença e com a rápida expansão desse nível de atenção por todo o país não implicou, necessariamente, uma mudança real do modelo assistencial em saúde bucal. Para os autores, algumas características inerentes à própria profissão conferem à saúde bucal particularidades que dificultam sua prática no setor público, como, por exemplo, a necessidade do uso de tecnologias duras na atenção básica.

Baldani et al. 1 também estudaram o processo de trabalho das ESB e concluíram que a incorporação das ações de odontologia, segundo a ESF, foi melhor nos municípios de pequeno porte, quando comparado aos de médio e grande portes, o que corrobora os resultados encontrados nesta pesquisa. Medina \& Aquino 29 explicam que, em municípios pequenos, altas coberturas populacionais podem ser obtidas com a instalação de um pequeno número de equipes, significando, para muitos deles, expansão dos serviços básicos. Enquanto, para os municípios maiores, a adoção da ESF exige um maior esforço de reorganização do sistema de saúde, pois, nesses municípios, já existia uma rede física instalada e um conjunto de profissionais atuando em uma outra lógica de organização dos serviços.

Não houve grandes diferenças no processo de trabalho das ESF quando comparadas com as equipes de atenção básica parametrizadas. O que não deveria ocorrer, visto que, segundo documento do Conselho Nacional de Secretários da Saúde (CONASS), a adoção da ESF deveria causar mudanças visíveis na forma como os serviços são ofertados, levando a um grande impacto nas condições de saúde da população 19. Porém, estudos como os de Bertoncini 30 e Costa et al. 31 têm apontado precárias condições de trabalho das equipes de saúde na ESF, sugerindo composição básica insuficiente das equipes; insuficiência de profissionais com o perfil proposto pelo programa; modalidades precárias de contrato de trabalho; e sobrecarga de atendimento. Como consequência, segundo os autores, há dificuldades em efetuar o planejamento e discutir a dinâmica do trabalho. Isso dificulta o desenvolvimento das práticas centradas nos atributos da atenção primária à saúde. Assim, o modelo assistencial na ESF fica semelhante ao de uma unidade de atenção básica tradicional, centrada no modelo assistencial biomédico.

Conduzir uma avaliação das conquistas do SUS nesses mais de vinte anos de sua criação não é tarefa fácil, em razão da complexidade e do tamanho da rede instalada. Todavia, é possível apontar avanços em várias áreas. Com este trabalho, ficou evidente que os atributos da atenção primária à saúde estão parcialmente incorporados ao processo de trabalho das ESB. 
Vale destacar que os dados do presente estudo se referiram somente às ESB que aderiram ao ciclo I do PMAQ-AB e não ao universo das equipes do país, o que pode não demonstrar a real situação do processo de trabalho nas ESB no Brasil. Desse modo, uma das principais limitações deste estudo se refere à amostra não probabilística, voluntária e desproporcional entre os estados brasileiros, variando de 6,7\% das equipes do Maranhão a 79,5\% em Santa Catarina. É esperado que essa adesão tenha se dado especialmente pelas equipes mais bem estruturadas. Tais características da amostra podem ter gerado viés de seleção nos resultados do estudo, provavelmente superestimando a qualidade do processo de trabalho, sobretudo nos locais com menor adesão. No entanto, os resultados, aqui, são apresentados de forma desagregada apenas para o nível das cinco macrorregiões; e incluem mais de $50 \%$ das equipes brasileiras, constituindo-se, portanto, no estudo com o maior tamanho amostral no país sobre essa temática. Outra fragilidade se refere à não disponibilidade de informações sobre as perdas amostrais detalhadamente para cada tipo de equipe. Dessa maneira, as perdas referidas - 280 equipes - não são específicas para as ESB. Contudo, mesmo se todas as perdas tivessem sido de ESB, isso representaria menos de 2,5\% do total das ESB que aderiram ao programa; logo, com pouca probabilidade de viés.

Diante da necessidade de incorporar a avaliação ao cotidiano dos serviços de saúde na atenção básica 15,16,22 e da importância de instituir um modelo assistencial fundamentado na atenção primária à saúde 5,15,19, são necessários instrumentos capazes de aferir os atributos da atenção primária à saúde 5,18. O PMAQ-AB representa uma potente ferramenta de gestão para induzir mudanças no processo de trabalho de forma a garantir que os serviços ofertados sigam esses princípios 32,33,34. Nesse contexto, o instrumento é válido para estimular a institucionalização da avaliação das atividades em saúde, induzindo reflexão contínua da equipe sobre o seu processo de trabalho. Essa postura reflexiva deve ser uma tendência da gestão contemporânea 33,34. Ademais, os resultados do presente estudo contribuem com a construção de evidências para justificar as mudanças necessárias no processo de trabalho das ESB e no próprio instrumento de avaliação externa do PMAQ-AB.

Os dados do processo de trabalho das ESB oriundos do ciclo I do PMAQ-AB conseguem mensurar três dos quatro atributos essenciais da atenção primária à saúde. Sugere-se acréscimo de perguntas no instrumento de coleta de dados do PMAQ-AB para melhor avaliar o atributo longitudinalidade. Por fim, há grande heterogeneidade no processo de trabalho das ESB no país e os atributos essenciais da atenção primária à saúde estão parcialmente incorporados ao processo de trabalho na atenção básica das ESB no Brasil. 


\section{Colaboradores}

D. M. Fagundes contribuiu na redação do manuscrito, análise dos dados, aprovação da versão final do artigo. E. B. A. F. Thomaz colaborou na concepção da proposta inicial do projeto, redação do manuscrito, análise dos dados, coordenação regional da coleta dos dados, revisão e aprovação da versão final do artigo. R. C. S. Queiroz contribuiu na concepção da proposta, coordenação da coleta dos dados, análise dos dados, revisão e aprovação da versão final do artigo. T. A. H. Rocha colaborou na concepção da proposta inicial do projeto, coordenação da coleta dos dados, análise dos dados, revisão e aprovação da versão final do artigo. N. C. Silva colaborou na concepção da proposta inicial do projeto, coordenação da coleta dos dados, análise dos dados, revisão e aprovação da versão final do artigo. J. R. N. Vissoci colaborou na análise dos dados, revisão e aprovação da versão final do artigo. M. C. M. Calvo contribuiu na concepção da proposta inicial do projeto, coordenação regional da coleta dos dados, revisão e aprovação da versão final do artigo. L. A. Facchini colaborou na concepção da proposta inicial do projeto, coordenação geral do projeto, coleta dos dados, revisão e aprovação da versão final do artigo.

\section{Agradecimentos}

Ao Departamento de Atenção Básica do Ministério da Saúde; a todos os pesquisadores e instituições de ensino e pesquisa que coordenaram e executaram a coleta dos dados, em especial ao consórcio liderado pela Universidade Federal de Pelotas (UFPel); à Fundação de Amparo à Pesquisa do Estado do Maranhão (FAPEMA) e ao Conselho Nacional de Desenvolvimento Científico e Tecnológico (CNPq) pelas bolsas de Produtividade em Pesquisa. Fundo Nacional de Saúde (avaliação externa, PMAQ-AB).

\section{Referências}

1. Baldani MH, Fadel, CB, Possamai T, Queiroz MGS. A inclusão da odontologia no Programa Saúde da Família no Estado do Paraná, Brasil. Cad Saúde Pública 2005; 21:1026-35.

2. Pereira MM, Viana LAL, Mestriner SF, Mestriner Júnior W. Uma reflexão sobre a inserção da saúde bucal na saúde da família. Investigação 2008; 8:97-104.

3. Starfield B. Primare care: balancing health needs, services and technology. New York: Oxford University Press; 1998.

4. Colussi CF, Calvo MCM. Avaliação da atenção em saúde bucal no Brasil: uma revisão de literatura. Saúde Transform Soc 2012; 3:92-100.

5. Cruz DB, Gabardo MCL, Ditterich RG, Moysés SJ, Nascimento AC. Processo de trabalho na estratégia saúde da família: uma perspectiva a partir da equipe de saúde bucal. Rev APS 2009; 12:168-75.

6. Nascimento AC, Moysés ST, Bisinelli JC, Moysés SJ. Oral health in the family health strategy: a change of practices or semantics diversionismo. Rev Saúde Pública 2009; 43:455-62.

7. Sanglard-Oliveira CA, Werneck MAF, Lucas SD, Abreu MHNG. Responsibilities of Oral Health Technician in the Family Health Strategy in Minas Gerais, Brazil. Ciênc Saúde Coletiva 2013; 18:2453-60.

8. Almeida G, Sarti FM, Ferreira FF, Diaz MDM, Campino ACC. Analysis of the evolution and determinants of income-related inequalities in the Brazilian health system, 1998-2008. Rev Panam Salud Publica 2013; 33:90-7.

9. Rocha RACP, Goes PSA. Comparison of access to Oral Health Services between areas covered and not covered by the Family Health Program in Campina Grande, Paraiba State, Brazil. Cad Saúde Pública 2008; 24:2871-80.

10. Pereira CRS, Patrício AAR, Araújo FAC, Lucena EES, Lima KC, Roncalli AG. Impacto da Estratégia Saúde da Família com equipe de saúde bucal sobre a utilização de serviços odontológicos. Cad Saúde Pública 2009; 25:985-96.

11. Soares FF, Figueiredo CRV, Borges NCM, Jordão RA, Freire MCM. Oral health teamwork in brazilian family healthcare strategy: an analysis of studies published between 2001 and 2008. Ciênc Saúde Coletiva 2011; 16:3169-80.

12. Pereira CRS, Roncalli AG, Cangussu MCT, Noro LRA, Patrício AAR, Lima KC. Impacto da Estratégia Saúde da Família sobre indicadores de saúde bucal: análise em municípios do Nordeste brasileiro com mais de 100 mil habitantes. Cad Saúde Pública 2012; 28:449-62.

13. Moura MS, Ferro FEFD, Cunha NL, Souza Nétto OB, Lima MDM, Moura LFAD. Saúde bucal na estratégia de saúde da família em um colegiado gestor regional do estado do Piauí. Ciênc Saúde Coletiva 2013; 18:471-80. 
14. Departamento de Atenção Básica, Secretaria de Atenção à Saúde, Ministério da Saúde. Gestão da atenção básica. Retratos da atenção básica. v. 3. Brasília: Ministério da Saúde; 2015.

15. Departamento de Atenção Básica, Secretaria de Atenção à Saúde, Ministério da Saúde. Política Nacional de Atenção Básica. Brasília: Ministério da Saúde; 2012. (Série E, Legislação em Saúde)

16. Souza DS. Gestão do trabalho das equipes de saúde bucal na atenção primária à saúde. In: Goes PSA, Moyssés SJ, organizadores. Planejamento, gestão e avaliação em saúde bucal. São Paulo: Artes Médicas; 2012.

17. Starfield B. Atenção primária: equilíbrio entre necessidades de saúde, serviços e tecnologia. Brasília: Organização das Nações Unidas para a Educação, a Ciência e a Cultura/Ministério da Saúde; 2002.

18. Oliveira MAC, Pereira IC. Atributos essenciais da atenção primária e a Estratégia Saúde da Família. Rev Bras Enferm 2013; 66:158-64.

19. Conselho Nacional de Secretários de Saúde. Atenção primária e promoção da saúde. Brasília: Conselho Nacional de Secretários de Saúde; 2007.

20. Cook KF, Kallen MA, Amtmann D. Having a fit: impact of number of items and distribution of data on traditional criteria for assessing IRT's unidimensionality assumption. Qual Life Res 2009; 18:447-60.

21. Fornell C, Larcker DF. Evaluating structural equations models with unobservable variables and measurement error. Journal of Marketing Research 1981;18,39-50.

22. Valentini F, Damásio BF. Average variance extracted and composite reliability: reliability coefficients. Psicol Teor Pesq 2016; 32:1-7.

23. Wang X, Kammerer CM, Anderson S, Lu J, Feingold $\mathrm{E}$. A comparison of principal component analysis and factor analysis strategies for uncovering pleiotropic factors. Genet Epidemiol 2009; 33:325-31.

24. Hee-Ju Kim. Common factor analysis versus principal component analysis: choice for symptom cluster research. Asian Nurs Res (Korean Soc Nurs Sci) 2008; 2:17-24.

25. Coordenação de Saúde Bucal, Departamento de Atenção Básica, Secretaria de Atenção à Saúde, Ministério da Saúde. Diretrizes da Política Nacional de Saúde Bucal. Brasília: Ministério da Saúde; 2004.
26. Departamento de Atenção Básica, Secretaria de Atenção à Saúde, Ministério da Saúde. Projeto SB Brasil 2003: condições de saúde bucal da população brasileira 2002-2003: resultados principais. Brasília: Ministério da Saúde; 2004. (Série C. Projetos, Programas e Relatórios).

27. Secretaria de Atenção à Saúde; Secretaria de Vigilância em Saúde, Ministério da Saúde. SB Brasil 2010 Pesquisa Nacional de Saúde Bucal: resultados principais. Brasília: Ministério da Saúde; 2011

28. Souza TMS, Roncalli AG. Saúde bucal no Programa Saúde da Família: uma avaliação do modelo assistencial. Cad Saúde Pública 2007; 23:2727-39.

29. Medina MG, Aquino R. Avaliando o Programa Saúde da Família. In: Sousa MF, organizador. Os sinais vermelhos do PSF. São Paulo: Editora Hucitec; 2002.

30. Bertoncini JH. Da intenção ao gesto - uma análise da implantação do Programa de Saúde da Família em Blumenau [Dissertação de Mestrado]. Florianópolis: Departamento de Saúde Pública, Universidade Federal de Santa Catarina; 2000.

31. Costa MBS, Lima CB, Oliveira CP. Atuação do enfermeiro no Programa de Saúde da Família (PSF) no Estado da Paraíba. Rev Bras Enferm 2000; 53:149-52.

32. Solla JJS, Santos FP. Avaliação da implantação das ações de controle, avaliação e auditoria na Gestão Plena do Sistema Municipal de Saúde. In: Ministério da Saúde, organizador. Experiências inovadoras no SUS: relato de experiências/gestão dos serviços de saúde. Brasília: Ministério da Saúde; 2002; p. 361-91

33. Felisberto E. Monitoramento e avaliação na atenção básica: novos horizontes. Rev Bras Saúde Matern Infant 2004; 4:317-21.

34. Felisberto E. Da teoria à formulação de uma Política Nacional de Avaliação em Saúde: reabrindo o debate. Ciênc Saúde Coletiva 2006; 11:553-63. 


\section{Abstract}

The study aimed to: (1) describe the work process in Brazil's oral health teams, based on the essential attributes of primary health care, according to geographic region, type of team, and the municipality's socioeconomic characteristics and (2) verify whether the data in the work process of the oral health teams in the Brazilian National Program to Improve Access and Quality in primary health (PMAQ-AB) were capable of measuring such attributes. This was a nationwide ecological study with data from cycle I of PMAQ-AB. The study included descriptive, exploratory factor, and confirmatory factor analyses $(\alpha=5 \%)$. Constructs were analyzed in light of the essential attributes of primary health care (first contact, coordination of care, comprehensiveness, and continuity). The first three constructs and a fourth factor were formed, called dental prosthesis actions. However, the continuity attribute was not formed. The models' goodness-of-fit measures were satisfactory. Factor loads were greater than 0.5 , except for the two variables in factor 3 . The actions most frequently performed by the oral health teams $(>60 \%)$ were in first contact, and the least frequent were those in comprehensiveness, highlighting referrals to specialties (7.6\%). There were differences in the work process in oral health teams between regions of the country, type of team, and certification strata $(p<$ $0.05)$. In conclusion, data on the work process in oral health teams from cycle primary health care in the services' work routine. Further research is recommended on continuity of care. In addition, the oral health teams participating in cycle I of $P M A Q-A B$ should make further progress in actions related to comprehensiveness and coordination of care.

Healthy Evaluation; Health Services; Primary Health Care; Oral Health

\section{Resumen}

Los objetivos de este trabajo fueron: (1) describir el proceso de trabajo de los equipos de salud bucal (ESB) en Brasil, conforme los atributos esenciales de la atención primaria a la salud, según regiones, tipo de equipo y características socioeconómicas de los municipios; además de (2) verificar si los datos del proceso de trabajo de las ESB en el Programa Nacional de Mejora del Acceso y Calidad de la Atención Básica (PMAQ-AB) fueron capaces de evaluar tales atributos. Es un estudio ecológico, de cobertura nacional, con datos del ciclo I del PMAQ-AB. Se realizaron análisis descriptivos, factoriales exploratorios y confirmatorios $(\alpha=5 \%)$. Los constructos creados se analizaron a la luz de los atributos esenciales de la atención primaria a la salud (primer contacto, coordinación del cuidado, integralidad y longitudinalidad). Se generaron los tres primeros constructos, y un cuarto factor, denominado acciones en prótesis dental. No obstante, el atributo longitudinalidad no se configuró. Las medidas de ajuste de los modelos fueron satisfactorias. Las cargas factoriales fueron mayores que 0,5, excepto en dos variables del factor 3. Las acciones más realizadas por las ESB (> 60\%) fueron las de primer contacto, y las menos comunes fueron las de integralidad, destacándose contar con referencias para especialidades (7,6\%). Hubo diferencias en el proceso de trabajo de las ESB entre las regiones, tipo de equipo y extracto de certificación $(p<0,05)$. Se concluye que los datos del proceso de trabajo de las ESB del ciclo I del $P M A Q-A B$ fueron capaces de discriminar tres de los cuatro atributos esenciales de la atención primaria a la salud en la rutina de los servicios. Se sugiere profundizar en la evaluación de la longitudinalidad. Además, las ESB participantes del ciclo I del PMAQ-AB necesitan avanzar en acciones relacionadas con la integralidad y coordinación del cuidado.

Evaluación em Salud; Servicios de Salud; Atención Primária de Salud; Salud Bucal
Recebido em 26/Mar/2017

Versão final reapresentada em 28/Fev/2018

Aprovado em 06/Abr/2018 